\author{
Military Technical College \\ Kobry El-Kobba \\ Cairo, Egypt
}

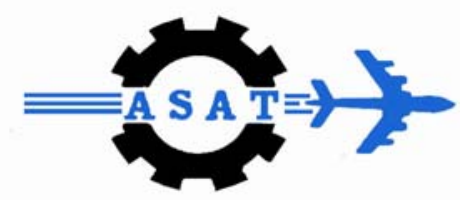

12-th International Conference

on

Aerospace Sciences \&

Aviation Technology

\title{
OPTIMIZATION AND PERFORMANCE LIMITS OF UNCOOLED INFRARED DETECTORS
}

\author{
Walid. A. Haggag and Ezz E. Farouk,
}

\begin{abstract}
Generally selecting of an IR detector for practical and commercial application, is difficult. We have to take into consideration several factors such as availability, cost, environmental stability and processing simplicity. These factors are often as important as radiometric performance and may well determine the final selection of a particular detector. Also, selection of the design parameters of a given detector is different according to the application. This work presents the ability to simulate the response of micro-devices using optimization analysis to give researchers insight of the behavior of devices before prototypes are developed as well as allowing the designer to optimize the desired response. We consider the main types of IR thermal detectors, their basic operational principles, and their performance parameters aiming to judge on their suitability for high speed thermal imaging applications. Mathematical models for the resistive bolometers, $\mathrm{p} n$ junction bolometers, pyroelectrics, are presented. Based on these mathematical models, the limiting factors of these detectors are identified, and their effect on the performance measures ( e.g., sensitivity and detectivity) is calculated. Unconstrained optimization techniques are then used to optimize the design parameters of an infrared thermal detector for the best detectivity. Next, constrained optimization techniques are used to evaluate the detectivity subject to a lower limit constraint on the frame rate. Our results indicate that the p-n junction diode bolometers offer the best detectivity at low frame rates $(30-60 \mathrm{~Hz})$ at room temperature, with a detectivity of $7 \times 10^{8} \quad(\mathrm{~cm} \sqrt{\mathrm{Hz}} / \mathrm{W})$. For high frame rate applications $(100-200 \mathrm{~Hz}$ ), the resistive bolometers offer the best detectivity at room temperature, with a detectivity of $6 \times 10^{8}(\mathrm{~cm} \sqrt{\mathrm{Hz}} / \mathrm{W})$.
\end{abstract}

\section{KEYWORDS:}

Uncooled Infrared, Thermal Detectors.

\section{INTRODUCATION}

Investigate the possibility of further improving the performance measures, specifically detectivity, by studying the effects of several design variables which has a great effect on the performance. To achieve this goal, the role of the deposition conditions, 
material resistivity, thermal conductance, bias voltage, layer thickness and device dimensions on the performance of the device, are analyzed.

Due to the increased complexity of the equations describing the detector responsivity, NEP, detectivity as well as the large number of parameters required to describe the system, a numerical solution is required. we investigate the effect of various parameters on the performance of resistive bolometers, $p-n$ junction and, pyroelectric detectors. The optimal value of each parameter is determined using optimization techniques. To compare, accurately the performance of different detectors the most frequently-used figure of merit is the detectivity. The optimal choice of these parameters is the goal of our discussion in this paper.

\section{Optimal Design Of The Resistive Bolometer}

The purpose of this section is to investigate the effects of some design parameters on the performance of a resistive bolometer. We choose the microbolometer structure with the CMOS n-well layer as the active element as shown in Fig. 1. This detector is based on the suspended n-well resistor, and is implemented in a $0.8 \mu \mathrm{m}$ CMOS process [2]. Infrared radiation heats the absorbing layer on the thermally isolated nwell, increasing its temperature, which in turn results in a change in its resistance related to its TCR. The bulk silicon under n-well is etched away to reduce thermal conductivity and to increase responsivity of the detector. This thermally isolated suspended structure is obtained by front-end bulk etching of fabricated CMOS dies in TMAH, while using the electrochemical etch-stop technique to prevent the etching of the n-well.

\section{Design Trade offs}

The performance of the n-well microbolometer pixel is affected by a number of design parameters and material characteristics, including interconnect layer on the support arms, pixel size, opening and support arm widths.

\section{Pixel Size}

Generally, the detection range of many uncooled IR imaging system is limited by pixel resolution. High resolution imaging requires small pixel size that implies reduced cost, and also reduced weight and size of the imager. In addition, the reduction in pixel size allows significantly larger number of FPAs (Focal Plane Arrays) to be fabricated on each wafer. Additionally, decreasing the pixel size decreases the thermal capacitance C,

$\mathrm{C}=\left(\right.$ heat capacity $\left(\mathrm{j} / \mathrm{cm}^{3} \mathrm{~K}\right) \times($ length $\times$ width $\times$ thickness $)$,

which in turn decreases the thermal time constant and enhances the performance. On the other hand, the pixel size cannot be decreased lower than a certain size determined by process design rules ( ncf. Fig. 2). As a design rule constraint, the openings cannot be made narrower than certain size if post-CMOS bulkmicromachining is to be used. Also, decreasing the pixel size causes decreasing the power incident on the active element so the detectivity will decrease. 


\section{Thermal Isolation (Thermal Conductance)}

The most important design parameter is the thermal isolation, because it determines the detectivity and thermal time constant of the device. Thermal conductance has a direct impact on responsivity [4] as shown in Eq. [1].

$$
\Re=\frac{\eta \alpha_{\text {pixel }}{ }^{\text {bias }_{\text {pixel }}{ }^{2}}}{G_{\text {th }} \sqrt{\left(1+(2 \pi f \tau)^{2}\right)}}
$$

It is clear that, the detector responsivity depends on the thermal conductance, $G_{t h}$, so in order to get high responsivity, we have to use the lowest possible value of $G_{\text {th }}$. Towards this goal, the support arms should be as long as possible and as thin as possible to enhance the responsivity. However, reducing the support $G_{t h}$ increases the device time constant, and reduces its ability to monitor rapid changes. For imaging FPAs, the thermal time constant must not exceed the inverse of the frame rate (if $\tau_{\max }=1 /(30 \mathrm{~Hz})=33 \mathrm{msec}$.). Thus, $G$ must be $\geq C / \tau_{\max }$. Also, very thin and long supports are mechanically fragile.

\section{Support Material}

The selection of the material of pixel supports has two effects on the performance. The first effect is related to the thermal isolation of the detector. The material used as an interconnect layer has to have low thermal conductivity. The second effect is related to the resistance of support arms which has a direct impact on the sensitivity of the detector. For sensitive operation of the detector, the resistance of the pixel should be much higher than the interconnect resistance. Also, high support resistance introduces large thermal noise to the device, and degrades its detectivity. We have two alternatives for interconnect layer material, metal and polysilicon. Use of metal layer as support arms provides a low electrical resistivity, thus, reduces the noise contribution and decreases the total resistance of pixel related to the Eq. [2].

$$
R_{\text {pixel }}=2 R_{\text {connect }}+R_{n-\text { well }}
$$

Yet, aluminum $(\mathrm{Al})$ has a poor thermal isolation due to its high thermal conductivity, $(181 \mathrm{~W} / \mathrm{mK})$. On the other hand, polysilicon layer has low thermal conductivity, (18 $\mathrm{W} / \mathrm{mK}$ ) [3], and high resistivity. Overall, the smaller $G_{t h}$ of the polysilicon material, rather than its high thermal noise and electrical resistance effects, renders it more favorable than the metal as a support material. Fig.3 and Fig.4 illustrate some of the trade-offs involved in the pixel design. In Fig. 3, we show the effect of changing the total pixel resistance on the noise voltage and detectivity. We note that increasing of the total pixel resistance, due to an increase of support resistance for example, causes an increase in the responsivity. However, increasing the pixel resistance also causes the pixel noise voltage to increase, and consequently degrades the detectivity as shown in Eqns [3] and [4].

$$
V_{n}=\sqrt{4 k T R_{\mathrm{det}} \Delta f_{e}}
$$




$$
D^{*}=\Re \frac{\sqrt{A_{D} \Delta f_{e}}}{V_{n}} \mathrm{~cm} \cdot H z^{1 / 2} W^{-1}
$$

Next consider the effect of the bias current. From the equation of the responsivity, there is a relationship between the bias current and responsivity as shown in Fig 5. As shown in Fig.5, the responsivity increases with increasing the bias current. Yet, increasing of bias current has a limit due to the self heating effect, which can affect the performance of the detector and damage the device.

\section{Formal Statement Of The Resistive Bolometer Optimization.}

We summarize our problem mathematically as follows:

Maximize $D^{*}$ Where the detectivity $D^{*}$ is function of $\left\{L_{a c}, W_{a c}, L_{s}, W_{s}, I_{b i a s}\right.$, $\left.N_{d}\right\}$. It is given by Eqn. [5]:

$D^{*}=\mathfrak{R} \frac{\sqrt{A_{D} \Delta f_{e}}}{V_{n}}$

(5)

The optimization search parameters $\left(L_{a c}, W_{a c}\right.$, ...etc.) from the unknown design vector are to be determined by the optimization process. The responsivity $\mathfrak{R}$ is given by Eqn. [6] :

$$
\mathfrak{R}=\frac{\eta \alpha_{\text {pixel }}{ }^{\text {bias }}{ }_{\text {pixel }}}{G_{\text {th }} \sqrt{\left(1+(2 \pi f \tau)^{2}\right)}}
$$

and the noise voltage $V_{n}$ is given by Eqn. [7].

$$
V_{n}=\sqrt{4 k T R_{\operatorname{det}} \Delta f_{e}}
$$

where $I_{\text {bias }}$ is the biasing current, $R_{\text {pixel }}$, is the total resistance of the pixel detector and is given by eqn. [8].

$$
R_{\text {pixel }}=2 R_{\text {connect }}+R_{n-\text { well }}
$$

(8)

Where $R_{n-\text { well }}$ is the resistance of active element and equals to, $R_{n-\text { well }}=\rho \frac{L_{a c}}{A_{D}}$ where $\rho$, is the resistivity of active element and is given by Eqn. [9]. 


$$
\rho=\frac{1}{q\left(n \times \mu_{n L i}+p \times \mu_{p L i}\right)}
$$

$n=N_{d}, \quad p=\frac{n_{i}^{2}}{N_{d}}$, where $N_{d}$ is the doping concentration and $n_{i}$ is the intrinsic carrier concentration and is given by Eqn. [10].

$n_{i}=3.9 \times 10^{16} \times T^{1.5} \exp \left(\frac{-0.605 \times 1.6 \times 10^{-19}}{k T}\right)$

(10)

where $\mu_{n L i}$ and $\mu_{p L i}$ are the mobility caused by lattice and ionized scattering for electrons and holes respectively and are given by:

$$
\begin{aligned}
& \mu_{n L_{i}}=\mu_{\min 1}+\frac{\left(\mu_{L 1}-\mu_{\min 1}\right)}{\left(1+\left(\frac{N_{d}}{C_{r e f n}}\right) \times \alpha\right)} \\
& C_{\text {refn }}=1.21 \times 10^{17} \mathrm{~cm}^{-3}\left(\frac{T}{300 \mathrm{~K}}\right)^{3.2} \\
& \mu_{p L_{i}}=\mu_{\min 2}+\frac{\left(\mu_{L 2}-\mu_{\min 2}\right)}{\left(1+\left(\frac{N_{a}}{C_{\text {refp }}}\right) \times \alpha\right)} \\
& C_{\text {refp }}=2.23 \times 10^{17} \mathrm{am}^{-3}\left(\frac{T}{300 \mathrm{~K}}\right)^{3.2} \\
& \mu_{L 1}=1430\left(\mathrm{~cm}^{2} / V s\right) \times\left(\frac{T}{300 K}\right)^{-2} \\
& \mu_{L 2}=460\left(\mathrm{~cm}^{2} / \mathrm{Vs}\right) \times\left(\frac{T}{300 K}\right)^{-2.18} \\
& \alpha=0.72 \times\left(\frac{T}{300}\right)^{0.065} \\
& \text { If } \mathrm{T} \geq 200 \mathrm{~K}
\end{aligned}
$$$$
\therefore \mu_{\min 1}=A 1 \times\left(\frac{T}{300 K}\right)^{-0.45} \text { and } \mu_{\min 2}=A 2 \times\left(\frac{T}{300 K}\right)^{-0.45}
$$$$
\therefore \mu_{\min 1}=\mathrm{A} 1 \times\left(\frac{200}{300}\right)^{-0.45} \times\left(\frac{\mathrm{T}}{200}\right)^{-0.15}
$$$$
\mu_{\min 2}=A 2 \times\left(\frac{200}{300}\right)^{-0.45} \times\left(\frac{\mathrm{T}}{200}\right)^{-0.15}
$$ 


\section{$A 1=80\left(\mathrm{~cm}^{2} / \mathrm{Vs}\right)$ for electrons and $A 2=45\left(\mathrm{~cm}^{2} / \mathrm{Ns}\right)$ for holes}

The thermal time constant $\left(\tau_{t h}\right)$ is given by Eqn.[11].

$$
\tau=\frac{C_{t h}}{G_{t h}}
$$

Where $C_{t h}$ is the thermal capacitance, given by Eqn.[12].

$C_{t h}=L_{a c} \times W_{a c} \times t_{a c}$

where $L_{a c}, W_{a c}$ and $t_{a c}$ are the length, width and thickness of the active element respectively. $A_{D}$ is the area of detector which is equal to $A_{D}=L_{a c} \times W_{a c}$. And $G_{t h}$ is the thermal conductance and is given by Eqn. [13]:

$$
G_{\text {th }}=g \frac{A_{S}}{L_{s}}
$$

Where $A_{S}$ is the cross sectional area of support and equal to $A_{s}=W_{S} \times t_{s}$. Where $t_{s}$, is the thickness of support and $g$, is the thermal conductivity of the support material. High frame rate performance constraint: $\tau<1 /$ frame rate. is enforced for the detectivity variation with the frame rate. Otherwise, this constraint is dropped.

\section{The Optimization Process Results For the N-Well Bolometer}

We used the optimization tool box of the Matlab program ( version 6.5) to solve the optimization problem defined in the last section. Table 1 gives the optimal values of the different design parameters of the design vector of the n-well microbolometer taking into consideration the design rules of the technology.

Another important point is considered in this paper, that is the suitability of each detector type for high frame rate IR sensors. Figure 6 gives the dependence of the detectivity on the frame rate for the n-well microbolometer by using constrained optimization techniques $[\tau<1 /$ frame rate].

\section{Optimal Design of The P-N Junction Diode Microbolometer.}

In order to implement a smaller pixels with a higher fill factor, a sub-micron $0.35 \mu \mathrm{m}$ CMOS process is considered for the $p-n$ junction bolometer. $p+$-active/n-well diode bolometers are designed using this process, and their performance is compared with the n-well resistor. In order to reduce the opening widths, the post-CMOS fabrication steps include an RIE- etching. It is possible to define openings between the support arms as narrow as $2 \mu \mathrm{m}$, allowing a reduction of the pixel size as shown in Fig. 7 [5]. As shown in Fig.7, infrared radiation heats the absorbing layer over the thermally isolated n-well, increasing its temperature, which in turn results in change in the diode voltage related to its temperature coefficient $\left(\frac{d V_{D}}{d T}\right)$. Bulk silicon under the $\mathrm{n}$-well is 
etched away to reduce thermal conductance and to increase responsivity of the detector. To obtain high thermal isolation, the interconnect material on support arms will be polysilicon. This thermally isolated suspended structure is obtained by front-side bulk etching, where the electrochemical etch-stop technique is used to prevent the etching of the n-well [5].

\section{Design Trade Offs}

The performance of $p-n$ junction diode bolometer is affected by a number of design parameters, such as the bias current. The sensitivity of the diode bolometer can be enhanced by decreasing the bias current and the doping impurity concentration. These parameters have to be optimized to give me the best detectivity of the detector.

\section{Bias Current}

For a constant bias current, it is clear that the detector voltage depends on the temperature ( see Fig.8). Observe that the rise in detector temperature results in a proportional decrease in the detector voltage at a constant forward current. The slope of temperature-voltage curve depends on the value of forward current.

Fig. 9 shows the I-V curve of the suspended diode type microbolometer detector without self heating effect.

As shown in Fig.9, the detector voltage increases with increasing bias current. This rather simple widely-known I-V diode characteristic assumes no self heating, or fixed temperature. If the self heating effect is taken into account, the $\mathrm{I}-\mathrm{V}$ characteristics of the diode looks like the one shown in Fig. 10.

As shown in Fig. 10, at low bias levels the diode forward voltage increases with increasing bias current, and self-heating is negligible at these low levels. However, at high bias currents, the slope of the I-V curve turns out to be negative. This behavior is due the fact that the applied bias causes high power dissipation inside the isolated $p-n$ diode bolometer, hence a significant increase in the detector temperature results, decreasing the detector voltage, as shown in Fig. 8. Also, increasing the bias current causes its temperature sensitivity to decrease in magnitude according to the Eqn [14]:

$$
\frac{d V_{\text {det }}}{d T}=\frac{d V_{D}}{d T}+R_{\text {connect }} I_{\text {bias }} \alpha_{\text {connect }}
$$

See also Fig.11. Consider now the effect of increasing the bias current on the detector noise voltage. Increasing the bias current results in a reduction of the dynamic resistance of diode according to:

$$
r_{d}=\frac{k T}{q . I_{\text {bias }}}
$$

Consequently, the noise voltage decreases with an increase in the bias current according to Eqn. [15].

$$
v_{n}=\sqrt{4 K T\left(R_{\text {connect }}+\frac{1}{2} r_{d}\right) \Delta f}
$$


Fig. 12 represents this noise behavior. It is clear from Fig.12 that the increase of bias current causes the responsivity to decrease, and at the same time reduces the noise voltage. Hence, the optimum detectivity is attained as a compromise between the poor noise performance at low bias currents, and the poor responsivity at high bias current. This detectivity behavior is shown in Fig. 13.

Note also that the performance or the detectivity is further degraded at high bias currents due to self-heating as shown in fig 14.

\section{Formal Statement Of The P-N Junction Diode Microbolometer}

We summarize our problem mathematically as follows:

Maximize the detectivity $D^{*}$ as function of $\left\{L_{a c}, W_{a c}, L_{s}, W_{s}, I_{b i a s}, N_{d}\right\} . D^{*}$ is given by : $\quad D^{*}=\mathfrak{R} \frac{\sqrt{A_{D} \Delta f_{e}}}{V_{n}}$

The optimization search parameters ( $L_{a c}, W_{a c}, \ldots$ etc.) form the unknown design vector are to be determined by the optimization process. The Responsivity $\mathfrak{R}$ is given by Eqn. [16].

$\Re=\frac{\eta}{G_{\text {th }}}\left(-\frac{d V_{D}}{d T}-\frac{R_{\text {connect }} I_{\text {bias }} \alpha_{\text {connect }}}{2}\right)$

where $\left(\frac{d V_{D}}{d T}\right)$ is the temperature sensitivity of the diode forward voltage and is used to compute the temperature sensitivity of the diode type detector $\left(\frac{d V_{\mathrm{det}}}{d T}\right)$. At constant bias current, the sensitivity of the diode type detector is given by Eqn.[17].

$$
\frac{d V_{\text {det }}}{d T}=\frac{d V_{D}}{d T}+R_{\text {connect }} I_{\text {bias }} \alpha_{\text {connect }}
$$

Where $\frac{d V_{D}}{d T}$ is derived as follows : $\quad \because \mathrm{I}=\mathrm{I}_{\mathrm{S}} \exp \left(\frac{q V_{D}}{n_{\text {if }} K T}\right)$, $V_{D}=\frac{n_{\text {if }} K T}{q} \ln \left(\frac{I}{I_{S}}\right)$

where $I_{S}$ is the reverse saturation current, $T$ is the junction temperature, and $K$ is the Boltzmann constant. The reverse saturation current of a long base diode is given by Eqn. [18]. 
$I_{S}=q n_{i} 2\left[\frac{D_{p}}{L_{p} N_{D}}+\frac{D_{n}}{L_{n} N_{A}}\right]$

where $n_{i}$ is the intrinsic carrier concentration, $D_{n}$ is the diffusion constants of electrons, $D_{p}$ diffusion constants of holes, $L_{p}$ is the diffusion length for holes, $L_{n}$ is the diffusion Length for electrons, $N_{D}$ is the Donors concentration of impurities and $N_{A}$ is the Acceptors concentration of impurities.

$L_{p}=\sqrt{D_{p}^{\tau} \tau_{p}} \quad, \quad L_{n}=\sqrt{D_{n} \tau_{n}} \quad, \quad D_{n}=\mu_{n} \cdot V_{t} \quad D_{P}=\mu_{p} V_{t}$ $\tau_{p}, \tau_{n}$ are the minority carrier lifetimes of holes and electrons respectively; $\mu_{p}$, $\mu_{n}$ are the mobility of holes and electrons respectively and $V_{t}$ is the thermal voltage $\left(V_{t}=\frac{K T}{q}\right)$. Both, diffusion constants and the lifetimes are temperature dependent. Intrinsic carrier concentration, $n_{i}$, which is strongly temperature dependent, is given by Eqn.[19]:

$$
n_{i}=\sqrt{N_{C} N_{V}} \exp \left(\frac{-E g}{2 k T}\right)
$$

Where ${ }{ }_{C}$ and $N_{V}$ are effective densities of states at the conduction-band and valence-band, respectively. The effective densities of states are given by :

$$
N_{C}=2\left(\frac{2 \pi m_{e} K T}{h^{2}}\right)^{3 / 2} \quad \text { and } \quad N_{V}=2\left(\frac{2 \pi m_{h} k T}{h^{2}}\right)^{3 / 2}
$$

$E_{g}(T)=E(0)-\left(\frac{\alpha T^{2}}{\beta+T}\right) \quad$ where, for silicon,

$E(0)=1.17 \mathrm{eV}, \alpha$ is $4.73 \times 10^{-4} / \mathrm{K}^{2}$ and $\beta$ is $636 \mathrm{~K} . \mathrm{K}$ is defined as a Kelvin and is given by: $\quad \mathrm{E}_{\mathrm{g}}(\mathrm{T})=1.17-\left(\frac{\alpha \cdot \mathrm{T}^{2}}{\beta+\mathrm{T}}\right) \quad$ and $\quad \frac{d \mathrm{E}_{\mathrm{g}}}{d T}=-\frac{\alpha T(2 \beta+T)}{(\beta+T)^{2}}$

$\therefore \frac{d V_{D}}{d T}=\frac{q V_{D}-E}{q T}-\frac{1}{q} \frac{d E g}{d T}-\frac{3 K}{q}$

The noise voltage is given by Eqn.[20].:

$v_{n}=\sqrt{4 K T\left(R_{\text {connect }}+\frac{1}{2} r_{d}\right) \Delta f}$ 
Where $r_{d}$ is the dynamic resistance of the diode and is given by $r_{d}=\frac{k T}{q \cdot I_{\text {bias }}}$

Using MATLAB optimization tool box, we solved this optimization problem to get the best optimized parameters of $p-n$ junction diode microbolometer. Table 2 lists these optimized parameters. The Design rules of the technology is taken into consideration during this optimization process.

Constrained optimization techniques are used to evaluate the detectivity subject to a lower limit constraint on the frame rate for $p-n$ junction diode microbolometer by using the constrain of $\left(\tau_{\max }<1 /\right.$ frame rate). Figure 15 gives the dependence of detectivity on the frame rate for the $\mathrm{p}-\mathrm{n}$ junction diode microbolometer.

\section{Comparison Of Results Of N-Well And The P-N Junction Bolometers}

It is clear from the Figs. 6 and 15, that our results indicate that the p-n junction diode bolometers offer the best detectivity at low frequency $(30 \mathrm{~Hz}-60 \mathrm{~Hz})$ at room temperature, with a detectivity of $7 \times 10^{8}(\mathrm{~cm} \sqrt{\mathrm{Hz}} / \mathrm{W})$. For high frame rate applications at the frequency range $100-200 \mathrm{~Hz}$, the resistive bolometers offer the best high detectivity at room temperature, with a detectivity of $6 \times 10^{8}(\mathrm{~cm} \sqrt{\mathrm{Hz}} / \mathrm{W})$. Fig. 16 sums up the detectivity - frame rate relationship for the two bolometers.

\section{Optimal Design Of The Pyroelectric Detector}

\section{Design Trade Offs}

Generally the desirable characteristics of a pyroelectric detector are high Curie temperature, large pyroelectric coefficient, large resistance, low loss tangent, and small heat capacity. The figure of merit $F_{D}$ of the pyroelectric materials is defined as:

$F_{D}=\frac{p}{C \sqrt{\varepsilon_{o} \varepsilon \tan \delta}}$

where $p$ is the pyroelectric coefficient, $C$ is the specific heat, $\varepsilon_{O}$ is the permittivity of vacuum, $\varepsilon$ is the dielectric constant and tan $\delta$ is the loss tangent. In optimizing the material characteristics; the dielectric constant, dielectric loss, and pyroelectric coefficient must all be considered together. Unfortunately an increase of $p$ is usually accompanied by an increase of dielectric constant, so that the figure of merit does not vary widely from one material to another. It is clear from this definition of $F_{D}$ that if we want to get high performance, we have to use a material of high Curie temperature and high pyroelectric coefficient. The pyroelectric detector is a little more difficult to deal with conceptually. The spontaneous polarization of pyroelectric material is maximum at low temperatures and changes slowly until the Curie temperature is approached where the polarization falls rapidly toward zero as shown in fig. 17 .

As shown in Fig.17, maximum sensitivity of a pyroelectric detector then is achieved by operating it at a temperature close to the Curie temperature but, of course still below it. The problem is that the response is very non-linear in this region. The trade-off is 
reached by choosing a pyroelectric material where the expected detector ambient or operating temperature is above the flat or non-responsive region but still not too close to the Curie temperature. Then the detector will have reasonable response that will be fairly close to linear at least for small temperature changes. It seems to be difficult to use a material of high pyroelectric coefficient, low dielectric constant and at the same time it has high Curie temperature. Perovskite thin films based on PZT have been reported to be a good choice. The fundamental performance parameter of a pyroelectric detector is the voltage responsivity which is defined as the ratio of output voltage induced by the pyroelectric effect due to the incident radiant power. The responsivity $\mathfrak{R}$ of the pyroelectric pixel is given by Eqn.[21] :

$\Re=\frac{V_{o}}{P_{o} A_{D}}=\frac{\eta \beta p A_{D} \omega R_{p}}{G_{t h}\left(1+\omega^{2} \tau_{t h}^{2}\right)^{1 / 2}\left(1+\omega^{2} \tau_{E}^{2}\right)^{1 / 2}}$

The characteristic thermal time constant $\tau_{\text {th }}$ of all thermal detectors, usually occurs at frequencies near $1-100 \mathrm{~Hz}$. The high frequency roll-off is determined by the electrical time constant $\tau_{E}$ of the detector load circuit. The bandwidth of the detector can be increased by decreasing the load resistance $R_{d}$ with a corresponding loss of voltage responsivity. For a maximum $\Re$, the impedance of the detector-load circuit should be maximum, and this is limited by the capacitance and dielectric loss of the crystal itself. Hence, there is a tradeoff between responsivity and temporal bandwidth. Fig.18, gives the dependence of the responsivity $(\omega)$ on the load resistance.

\section{Formal Statement Of Pyroelectric Detector}

We summarize our problem mathematically as follows:

Maximize the detectivity $D^{*}$ as a function of $\left\{L_{a c}, W_{a c}, L_{s}, W_{S}, R_{d}, \omega, D^{*}\right.$ is given by : $\quad D^{*}=\mathfrak{R} \frac{\sqrt{A_{D} \Delta f_{e}}}{V_{n}}$

The optimization search parameters $\left(L_{a c}, W_{a c}, \ldots\right.$...etc.) form the unknown design vector to be determined by the optimization process. The responsivity $\Re$ can be found by dividing the output voltage by the optical power, as shown below. The responsivity $\Re$ of the pyroelectric pixel is given by: $\quad \mathfrak{R}=\frac{V_{O}}{P_{O} A_{D}}$

where $P_{o}$ is the maximum irradiance in $\left(\mathrm{W} / \mathrm{m}^{2}\right)$ incident uniformly on the surface of a pixel area $\left(A_{D}\right.$ and thickness $\left.t_{a c}\right)$. The incident radiation $\mathrm{P}(\mathrm{t})$ is assumed to be sinusoidally modulated at a frequency $\omega$, thus, $P(t)=P_{O} A_{D} \exp (j \omega t)$. For the equivalent circuit for a pyroelectric detector shown in Fig 19. The short circuit signal 
current $i_{S}$ flowing through the parallel resistance $R_{p}$ generates a signal voltage $V_{S}$ at the input to amplifier is given by Eqn.[22]:

$$
V_{o}=i_{S} Z=i_{S} \frac{R_{p} \frac{1}{j \omega C_{p}}}{R_{P}+\frac{1}{j \omega C_{p}}}
$$

The output of a pyroelectric pixel is coupled to the input of an amplifier characterized by an input resistance $R_{A}$ and input capacitance $C_{A}$. Therefore, the parallel resistance $R_{p}$ is given by: $\quad R_{p}=\frac{R_{A} R_{d}}{R_{A}+R_{d}}$

Also, the total parallel capacitance $C_{p}$ equals the sum of $C_{d}$ and the input capacitance of amplifier $C_{A}$. Usually the input capacitance $C_{A}$ can be neglected with respect to the pixel capacitance $C_{d}$.

$V_{o}=i_{S} \frac{{ }_{p}}{\sqrt{1+R_{p}^{2} \omega^{2} C_{d}^{2}}}$

The current flows due to the change of polarization in time, so the current is given by:

$$
\begin{aligned}
& \because \Delta Q=P A_{D} \Delta T \\
& i_{S}=\frac{d(\Delta Q)}{d t}=p A_{D} \frac{d(\Delta T)}{d t} \\
& i_{s}=A_{D} \frac{d p}{d T} \times \frac{d T}{d t} \\
& \therefore \quad i_{s}=A_{D} p \frac{d T}{d t}
\end{aligned}
$$

Where $p$ is the pyroelectric coefficient, then

$$
d T=\Delta T e^{j \omega t} \quad \omega=2 \pi f \quad \frac{d T}{d t}=j \omega \Delta T e^{j \omega t}=j \omega d T
$$

$i_{s}=\omega p A_{D} \Delta T$

The temperature change $\Delta T$ can be derived from the following thermal heat balance equation, $\quad \eta \beta A_{D} P(t)=C_{t h} d \frac{\Delta T}{d t}+G_{t h} \Delta T$

Where $\eta$ is the sensor absorptivity. Let a thermal time constant $\tau_{t h}=\frac{C_{t h}}{G_{t h}}$. Under this condition of steady state, the solution for

$$
\Delta T=\frac{\eta \beta A_{D} P_{o} \exp (j \omega t)}{G_{t h}+j \omega C_{t h}}
$$

The RMS value of $\Delta T$ is given by Eqn.[23]: 


$$
\Delta T=\frac{\eta \beta A_{D} P_{o}}{G_{t h}\left(1+\omega^{2} \tau_{t h}^{2}\right)^{1 / 2}}
$$

The RMS amplitude of current is given by Eqn.[24]:

$i_{s, r m s}=\frac{\eta \beta p A_{D}{ }^{2} \omega P_{o}}{G_{t h}\left(1+\omega^{2} \tau_{t h}^{2}\right)^{1 / 2}}$

The pyroelectric pixel is characterized by a loss resistance $R_{d}$, related to the loss tangent $\left(\tan \delta\right.$ ) by Eqn.[25]: $R_{d}=\frac{1}{\omega C_{d} \tan \delta}$

The pixel loss resistance and capacitance together define an electrical response time, $\tau_{E}$ given which is given by $\tau_{E}=R_{d} C_{d}$

Then, $\tau_{E}=\frac{1}{\omega \tan \delta}$

$\because \mathrm{V}_{0}=i_{S} \times Z$

$$
V_{o}=\frac{\eta \beta p A_{D}{ }^{2}{ }^{2} P_{o} R_{p}}{G_{t h}\left(1+\omega^{2} \tau_{T}{ }^{2}\right)^{1 / 2}\left(1+\omega^{2} \tau_{E}{ }^{2}\right)^{1 / 2}}
$$

Thus the responsivity $\mathfrak{R}$ of the pyroelectric pixel is given by Eqn.[26] :

$\mathfrak{R}=\frac{V_{o}}{P_{O} A_{D}}=\frac{\eta \beta p A_{D} \omega R}{G_{t h}\left(1+\omega^{2} \tau_{t h}^{2}\right)^{1 / 2}\left(1+\omega^{2} \tau_{E}^{2}\right)^{1 / 2}}$

Typically the electrical time constant $\tau_{E} \ll<$ the thermal time constant $\tau_{t h}$. Thus, at low modulation frequencies $\left(f<\frac{1}{\tau_{\text {th }}}\right)$, the responsivity increases with the frequency due to the factor $\omega$ in the numerator. For the intermediate frequency range, $\frac{1}{\tau_{\text {th }}}<f<\frac{1}{\tau_{E}}$, the responsivity is almost flat as shown in Fig. 20. For $f>1 / \tau_{E}$, the responsivity drops with further $f$ increase. The Johnson noise (Dielectric loss noise) is usually the dominant noise source in pyroelectrics. In capacitive materials, including pyroelectric materials, Johnson noise is associated with the loss resistance.

$$
V_{J}=\sqrt{4 K T R_{d} \Delta f}
$$

By computing noise voltage and responsivity we get,

$$
D^{*}=\frac{\mathfrak{R} \sqrt{A_{D} \Delta f}}{V_{J}}
$$


The detectivity behavior as function of $f$ is similar to that of the responsivity ( cf. Fig.21). In Fig.21, we note that the detectivity $D^{*}$, is maximum at $100 \mathrm{~Hz}$. We see that $D^{*}$ was proportional to $f^{-1 / 2}$ for $f>100 \mathrm{~Hz}$, because the responsivity $\mathfrak{R}$, varied proportional to $\Delta f^{-1}$ and $V_{n}$ when we substitute by the equations of noise voltage and the responsivity in the equation of the detectivity $D^{*}$.

\section{Optimization Results for The Pyroelectric Detector}

We used the optimization tool box of the Matlab program ( version 6.5) ( Mediumscale: SQP, Quazi-Newton, line-search) to solve the optimization problem defined in the last section. Table 3 gives the optimal values of the different design parameters of the design vector of the pyroelectric detector taking into consideration the design rules of the technology.

\section{Conclusion}

We have the ability to simulate the response of uncooled infrared thermal detectors by using optimization analysis to give us the behavior of devices before prototypes are developed as well as allowing us to optimize the desired response. Compare the performance of IR detectors in terms of the specific detectivity $D^{*}$, which defines the value inverted to minimum detectable power. Studying the effects of several design variables on the performance of the different type of thermal detector structures by using matlap optimization tool box. Our results indicate that the $p-n$ junction diode bolometers offer the best low frequency detectivity at room temperature, with a low frequency $(30-60 \mathrm{~Hz})$ and detectivity of $7 \times 10^{8}(\mathrm{~cm} \sqrt{\mathrm{Hz}} / \mathrm{W})$. For high frame rate applications, the resistive bolometers offer the best high frequency detectivity at room temperature, with a frequency $(100-200 \mathrm{~Hz})$ and a detectivity of $6 \times 10^{8}(\mathrm{~cm} \sqrt{\mathrm{Hz}} / \mathrm{W})$. Thus the optimization of infrared performance is a matter of great practical importance investigate the possibility of further improving the performance of our detector specially the detectivity $D^{*}$.

\section{References}

[1] Deniz S. Tezcan, Selim Eminoglu, Orhan S. Akar, Tayfun Akin, " An Uncooled Microbolometer Infrared focal Plane Array in Standard CMOS " Proceedings of SPIE Vol. 4288, pp. 112-121, (2001).

[2] S. Eminoglu, M.Y. Tanrikulu, and T. Akin, "Low-Cost Uncooled Infrared Detector Arrays in Standard CMOS," SPIE AeroSense Symposium, Infrared Technology and Applications XXIX (Aerosense 2003), SPIE Vol. 5074, pp. 425436, Orlando, Florida, April 21-25, 2003.

[3] D.S. Tezcan, F. Kocer, and T. Akin, " An Uncooled Microbolometer Infrared Detector in any Standard CMOS Technology ", The 10th Int. Conf. on Solid-State Sensors and Actuators (TRANSDUCERS'99), pp. 610-613, Sendai, Japan, June 7-10, 1999. 
[4] S. Eminoglu, D. Sabuncuoglu Tezcan, M.Y. Tanrikulu, and T. Akin, "A Low-Cost Uncooled Infrared Detectors in Standard CMOS Technology," IEEE Transaction on Electron Devices, Vol. 50, NO.2, February 2003.

[5] Selim Eminoglu, M. Yusuf Tanrikulu, Tayfun Akin, " Low-Cost Uncooled Infrared Detector Arrays in Standard CMOS " Infrared Technology and Application XXIX, proceedings of SPIE Vol. 5074, pp. 425-436 (2003).

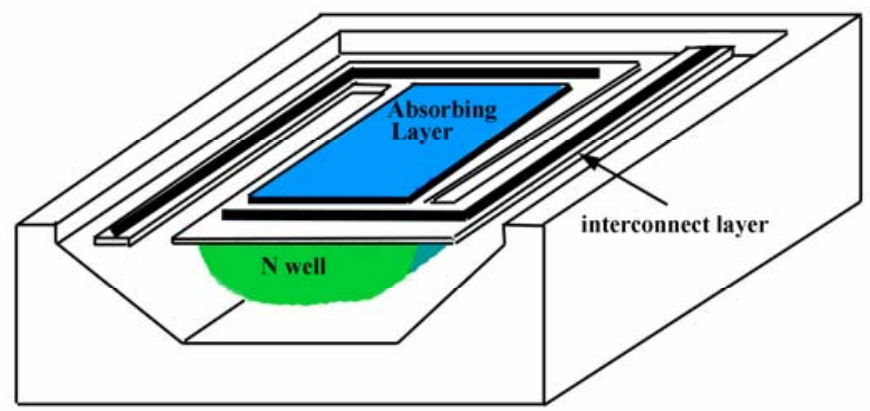

Fig. 1 Perspective view of n-well microbolometer [1]

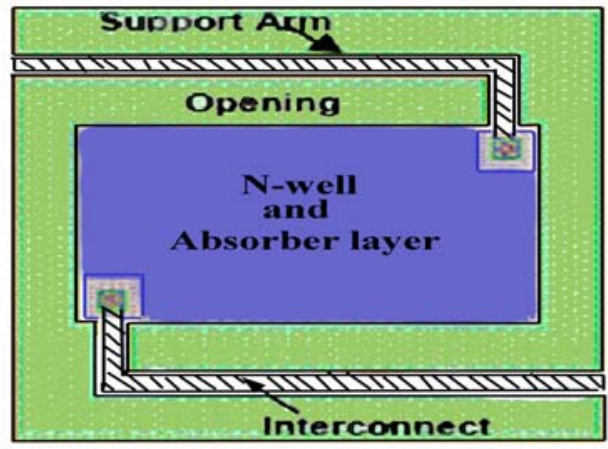

Fig. 2 Layout of an n-well microbolometer pixel [3].

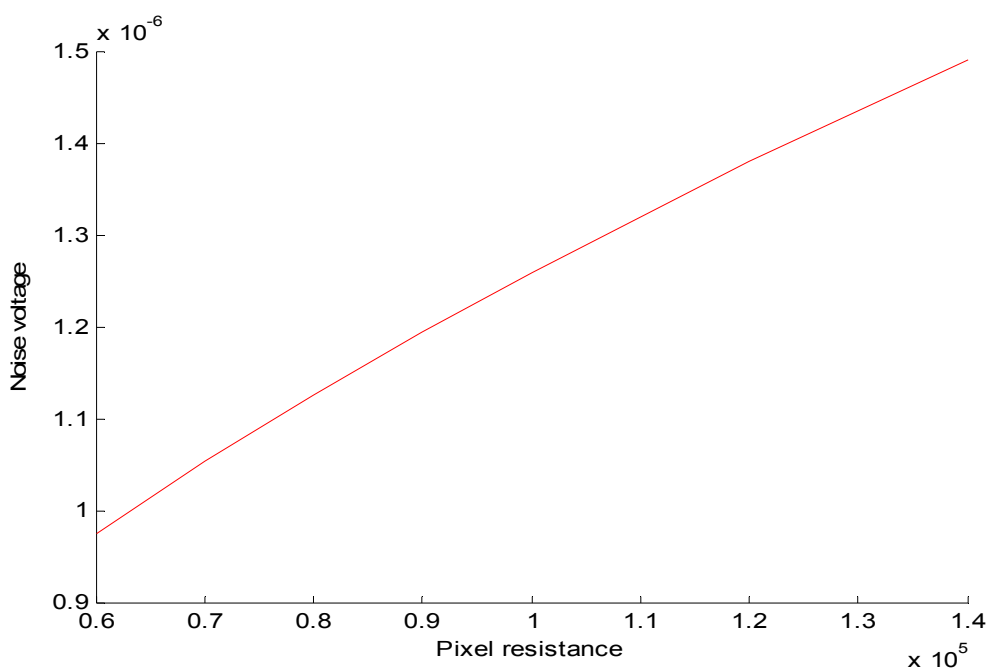

Fig. 3 The dependence of the noise voltage on the pixel resistance 


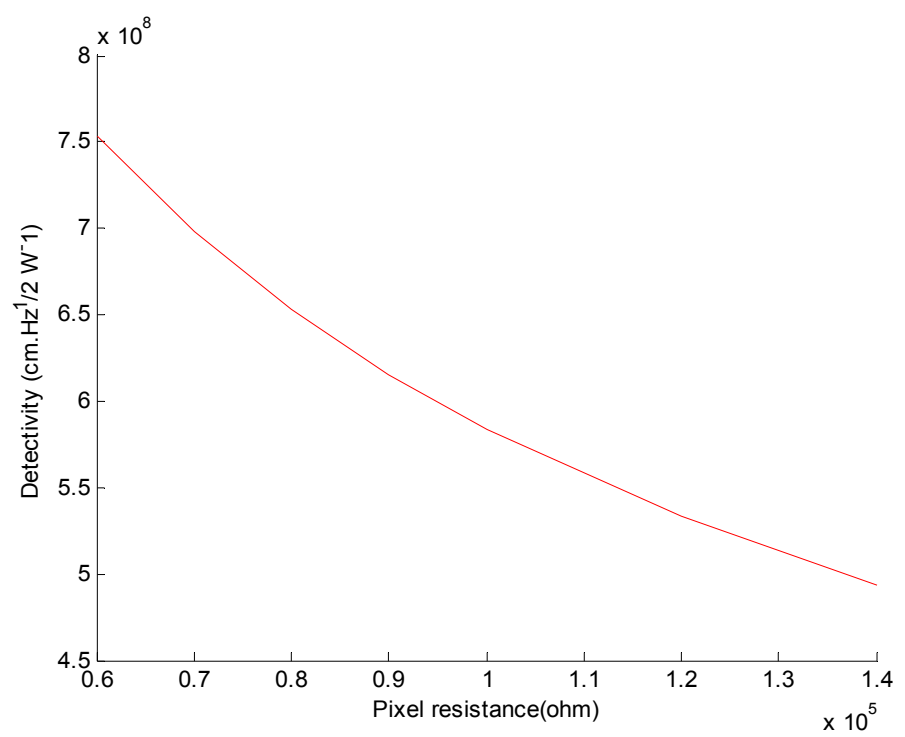

Fig. 4 The dependence of the detectivity on the pixel resistance

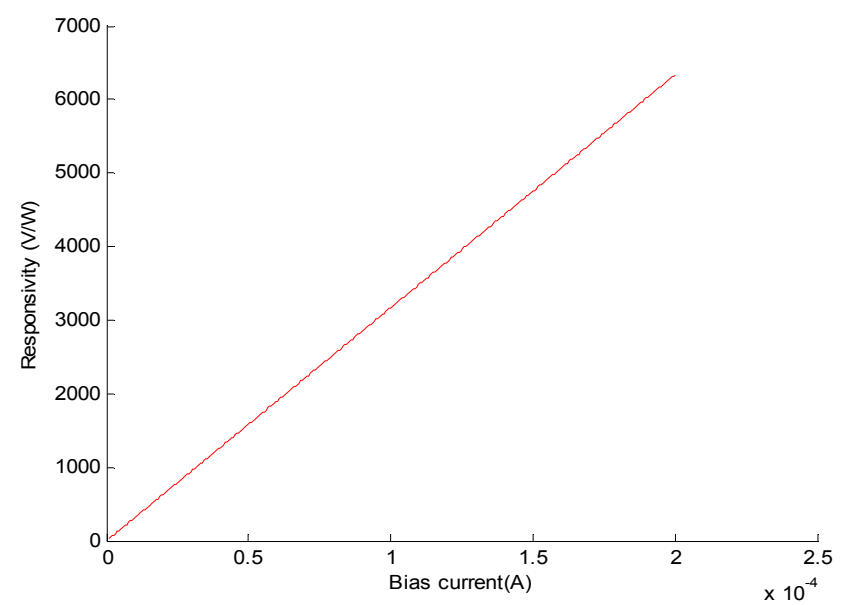

Fig. 5 Dependence of the responsivity on the bias current

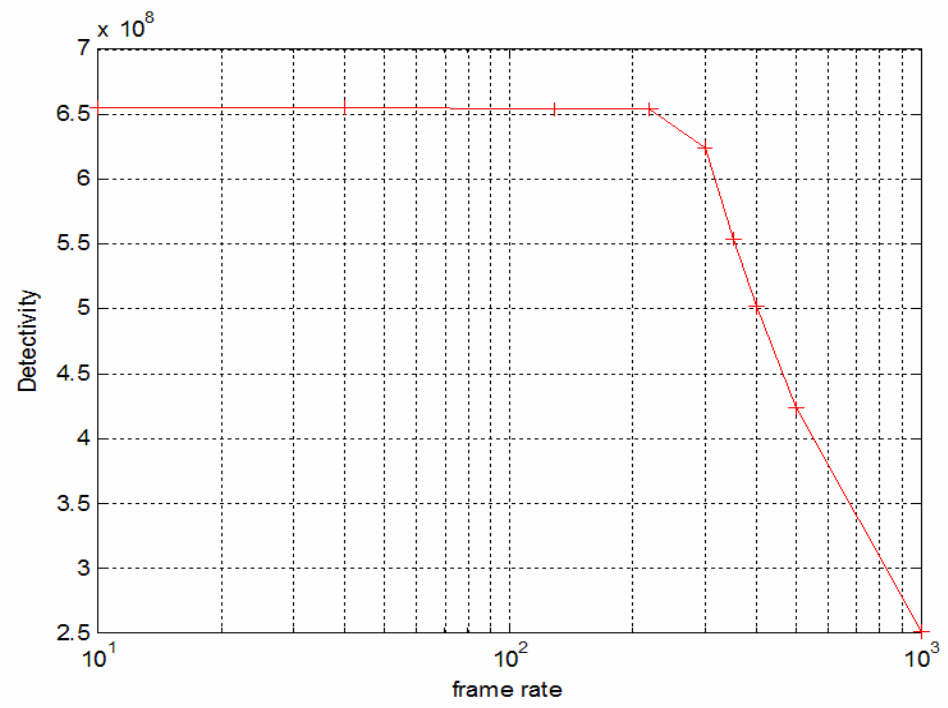

Fig. 6 Dependence of detectivity on the frame rate of $n$-well microbolometer 


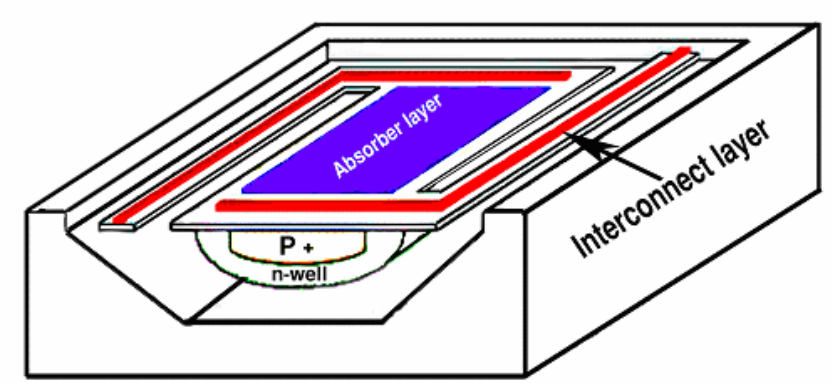

Fig. 7 The perspective view of the p+-active/n-well diode microbolometer that can be obtained in a standard n-well CMOS process [5].

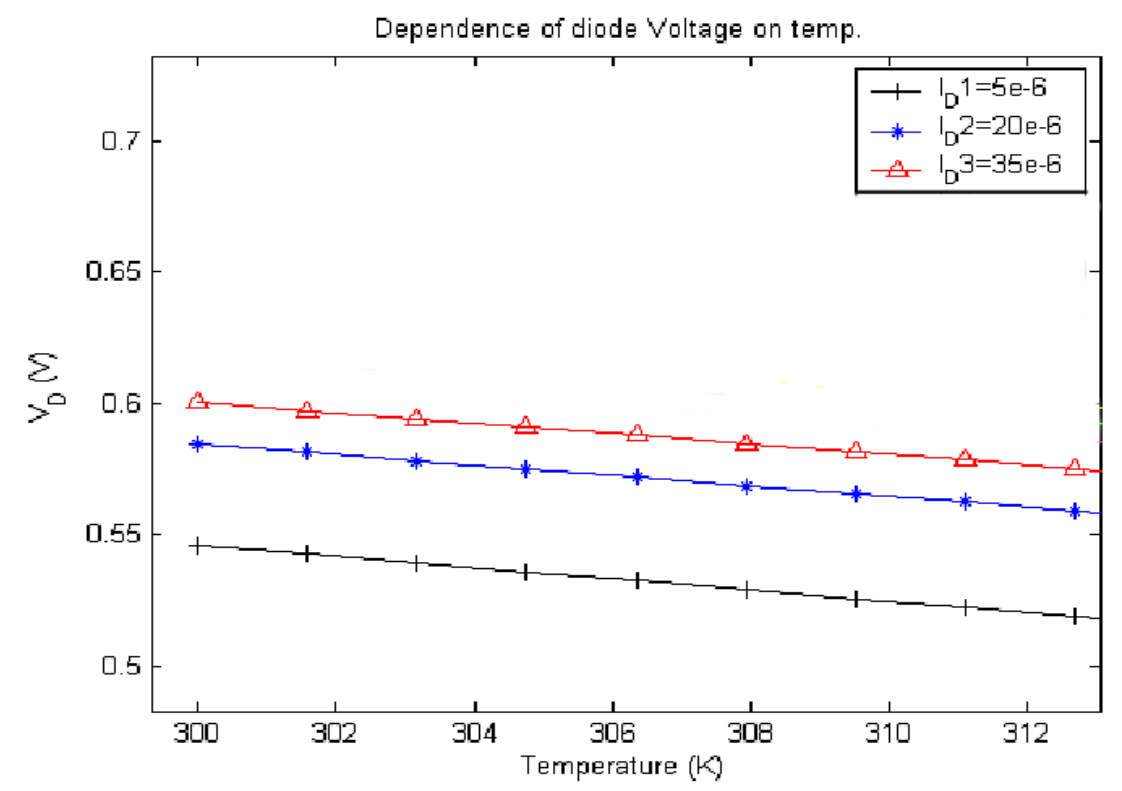

Fig. 8 Forward voltage versus temperature for different bias currents.

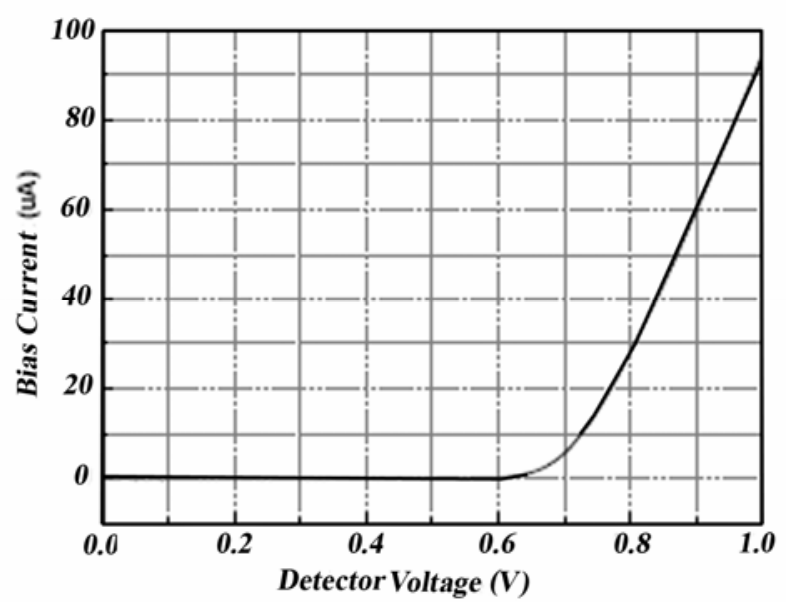

Fig 9 I-V curve of the suspended diode type microbolometer without self heating 


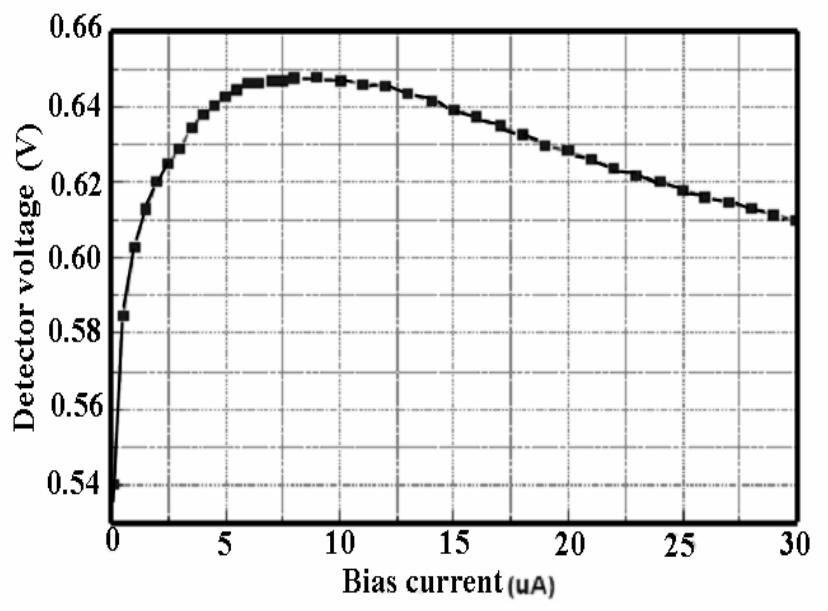

Fig.10 The relationship between bias current and detector voltage with self heating effect

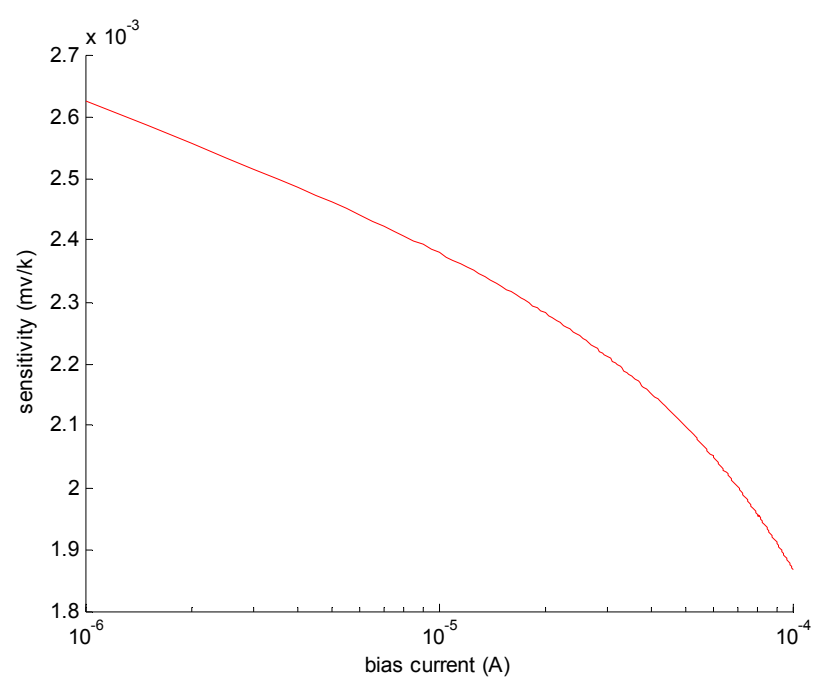

Fig. 11 Dependence of the absolute detector voltage temperature sensitivity with the pixel bias current

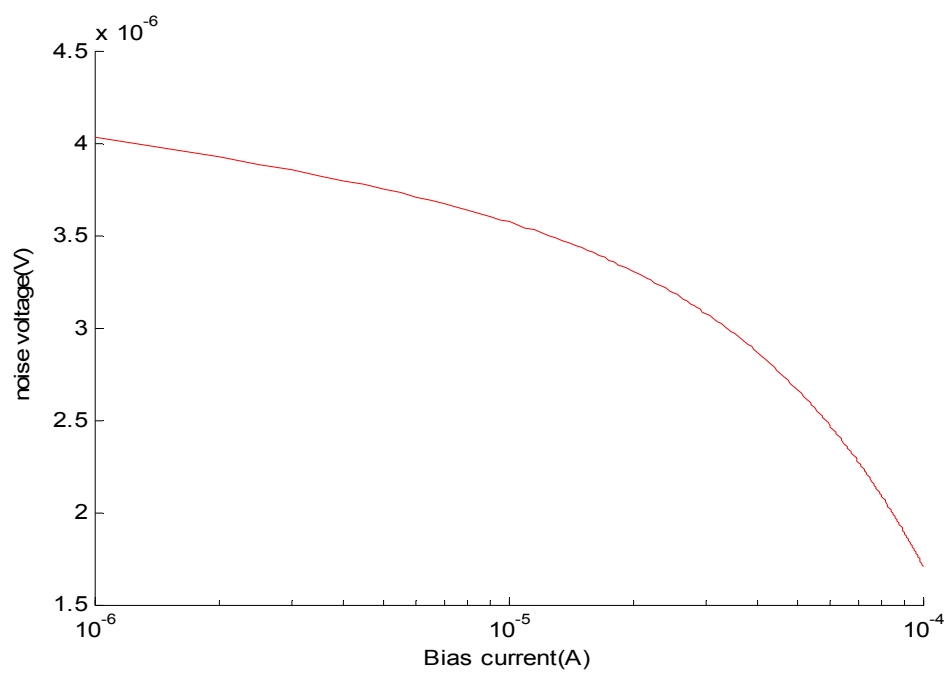

Fig.12 Output noise voltage dependence on the detector bias current 


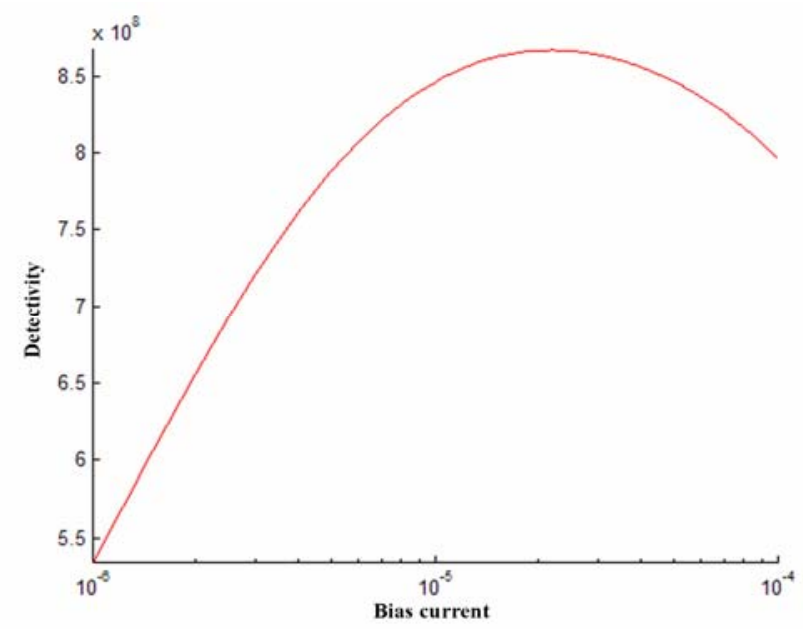

Fig.13 The dependence of the detectivity on the bias current

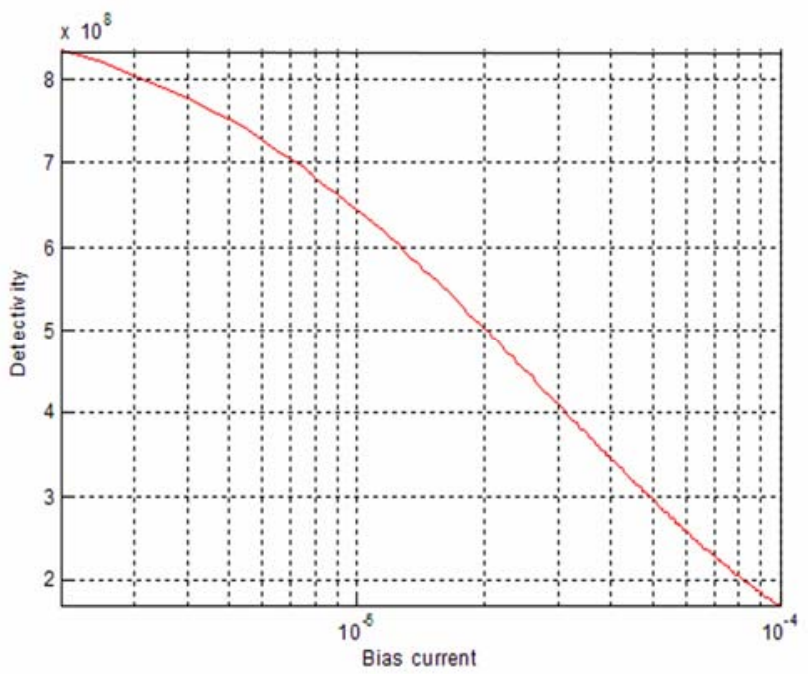

Fig.14 The dependence of the detectivity on the self-heating

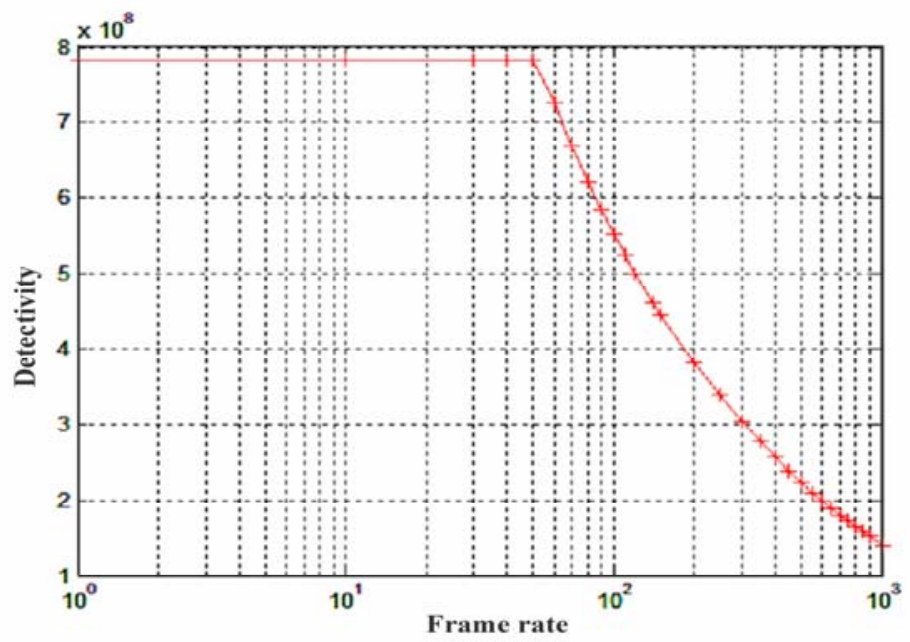

Fig.15 Dependence of detectivity on the frame rate of $\mathrm{P}-\mathrm{N}$ junction diode Bolometer 


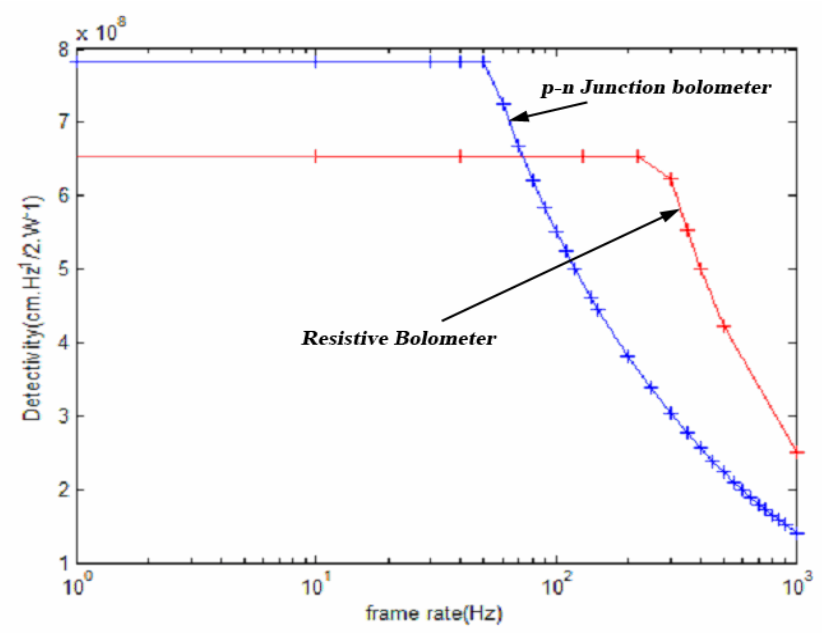

Fig. 16 Dependence of detectivity on the frame rate of $n$-well and $p-n$ junction microbolometer

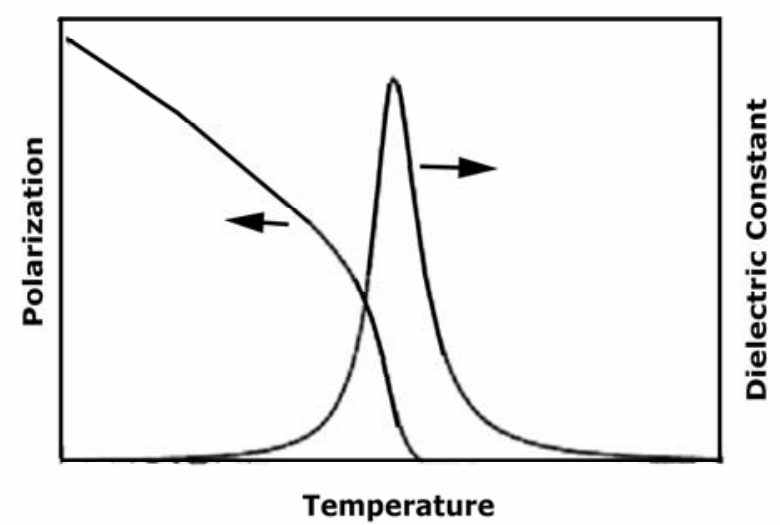

Fig.17 Temperature dependence of important pyroelectric properties near the phase transition

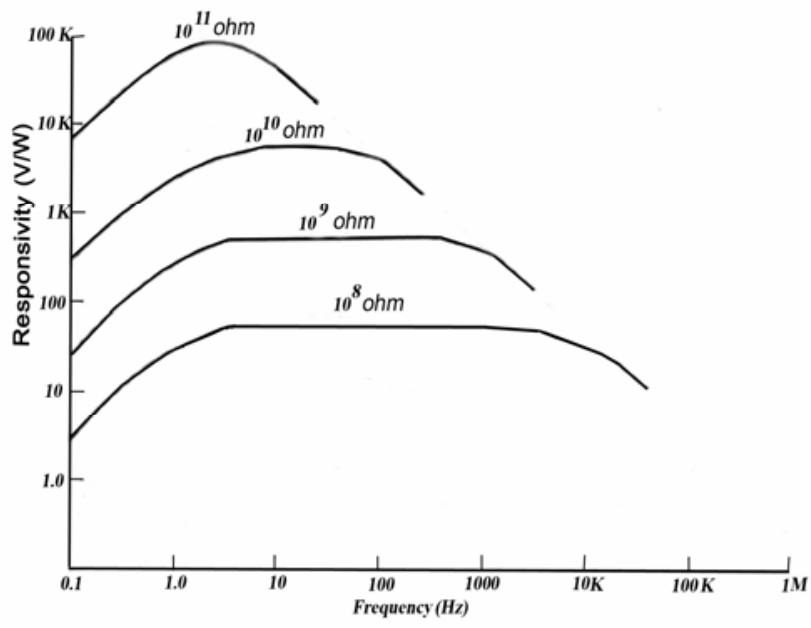

Fig.18 Responsivity variation with load resistance 


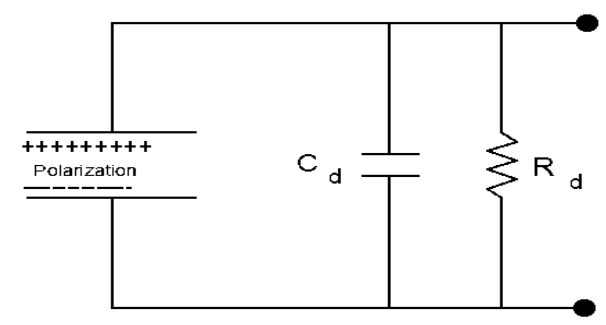

Fig.19 Equivalent circuit of pyroelectric detector

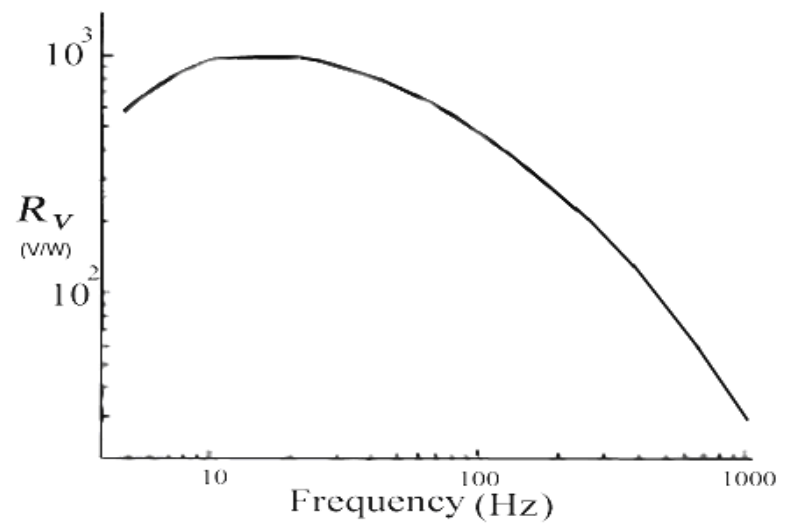

Fig.20 Dependence of the Responsivity on the modulation frequency

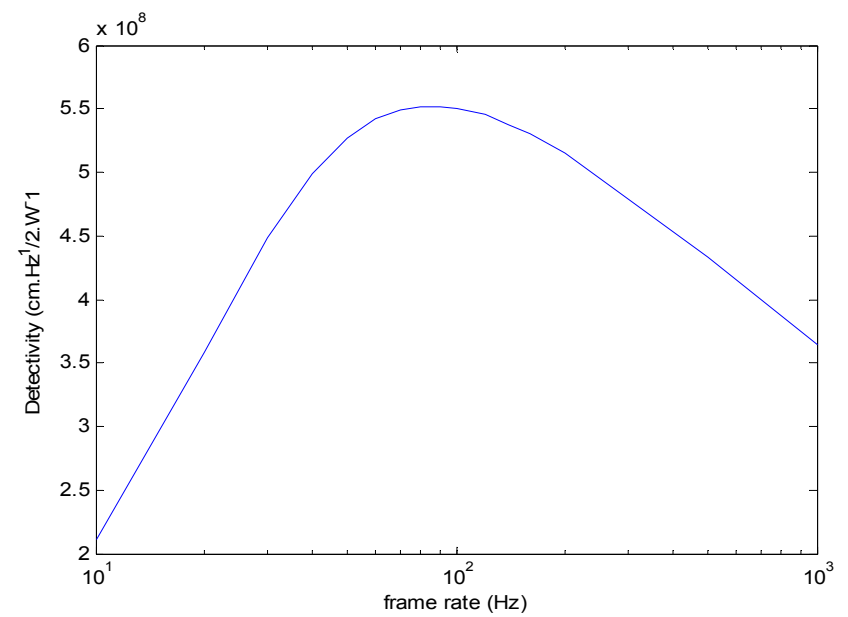

Fig.21 The dependence of detectivity on the frequency for a pyroelectric detector 
Table 1 Optimized parameters of $\mathrm{n}$-well microbolometer

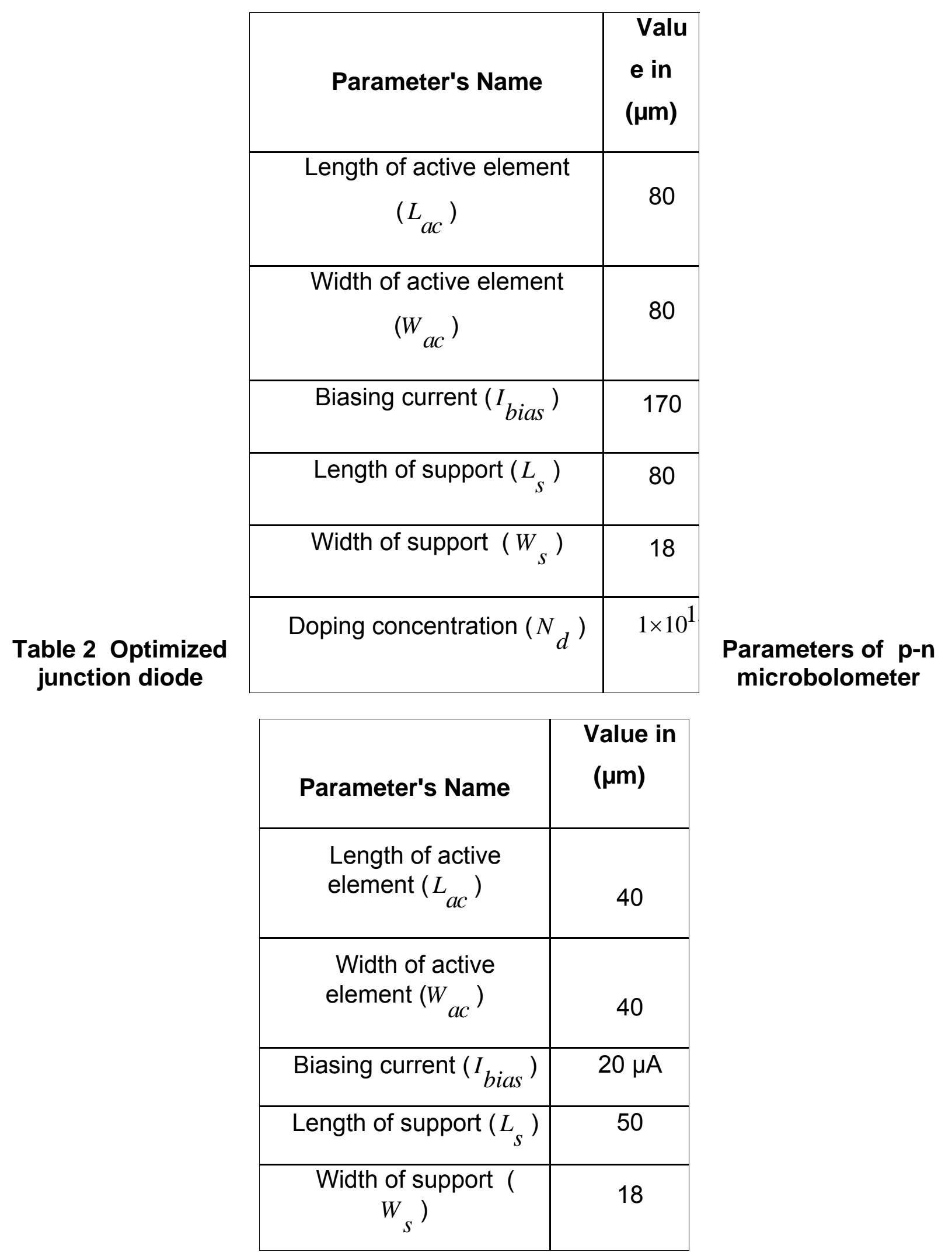




\begin{tabular}{|c|c|}
\hline $\begin{array}{c}\text { Doping concentration } \\
\left(N_{d}\right)\end{array}$ & $\begin{array}{l}1 \times 10^{17} \\
\mathrm{~cm}^{-3}\end{array}$ \\
\hline
\end{tabular}

Table 3 Optimized Parameters of pyroelectric detector

\begin{tabular}{|c|c|}
\hline Parameter's Name & $\begin{array}{c}\text { Value } \\
\text { in }(\boldsymbol{\mu m})\end{array}$ \\
\hline Length of active element $\left(L_{a c}\right)$ & 50 \\
\hline Width of active element $\left(w_{a c}\right)$ & 50 \\
\hline Pixel Capacitance $\left(C_{d}\right)$ & $10 \mu \mathrm{F}$ \\
\hline Length of support $\left(L_{S}\right)$ & 50 \\
\hline Width of support $\left(W_{S}\right)$ & 18 \\
\hline Frame rate $(\omega)$ & $50-60$ \\
\end{tabular}

